\title{
Link between Systemic and Cerebral Circulatory Levels of Microparticles and Necrosis Area of Stenotic Carotid Artery in the Patients Undergoing Carotid Stenting
}

\author{
Pei-Hsun Sung ${ }^{1}$, Meng-Shen Tong ${ }^{1}$, Chi-Hsiang Chu ${ }^{2}$, Fan-Yan Lee ${ }^{3}$, Hon-Kan Yip ${ }^{1,45,6,+}$
}

\begin{abstract}
Objective: We tested the hypothesis that (1) platelet-derived activated microparticles (PDAcMPs) and endothelial-derived activated (EDAC)-MPs were significantly higher after carotid stenting (CS) than before CS in carotid artery (CA) (defined as systemic circulation) and internal jugular vein (IJV) (defined as cerebral circulation) and (2) significantly correlated with necrotic area in CA stenosis measured by virtual histology intra-vascular ultrasound (VH-IVUS) prior to CS.
\end{abstract}

Methods: From September 2013 to 2016 July, a total of 92 consecutive patients undergoing CS were prospectively enrolled into the study. Blood samples for level of MPs were collected from CA and IJV prior to and 5-mintes after CS, respectively.

Results: The frequency of previous ischemic stroke (IS) was $45.7 \%$ (42/92). The mean stenosis prior to CS was $88.8 \%$. The procedural success was $100 \%$ and incidences of TIA and acute IS were $3.3 \%(3 / 92)$ and $4.3 \%(4 / 92)$, respectively. No significant correlation existed between severity of CA stenosis and circulating MPs level ( $p>0.5)$. The PDAc-MPs was significantly higher in post-CS procedure than in pre-CS procedure in both RIJV and CA (all $p<0.007$ ), whereas the EDAc-MPs was significantly higher in post-CS procedure than in pre-CS procedure in CA $(p=0.003)$. Post-CS PDAc-MPs and EDAc-MPs as well as ratio of post- to pre-CS PDAc-MPs were moderately correlated to necrotic area of carotid plagues (all $p<0.005$ ).

Conclusion: Cerebral and systemic PDAc-MPs and systemic EDAc-MPs were markedly increased in post CS than in that of prior to CS. Post-CS PDAc-MPs/EDAc-MPs and ratio of post/pre-CS PDAc-MPs were notably correlated to necrotic area of stenotic carotid plaque.

\section{Keywords}

Platelet-derived activated microparticles, Endothelial-derived activated microparticles, Carotid stenting, Necrotic area, Right internal jugular vein

\footnotetext{
'Division of Cardiology, Department of Internal Medicine, Kaohsiung Chang Gung Memorial Hospital and Chang Gung University, College of Medicine, Kaohsiung, 83301, Taiwan

${ }^{2}$ Clinical Trial Center, Kaohsiung Chang Gung Memorial Hospital, 83301, Taiwan

${ }^{3}$ Division of Cardiovascular surgery, Department of Surgery, Kaohsiung Chang Gung Memorial Hospital and Chang Gung University, College of Medicine, Kaohsiung, 83301, Taiwan

${ }^{4}$ Institute for Translational Research in Biomedicine, Center for Shockwave Medicine and Tissue Engineering, Kaohsiung Chang Gung Memorial Hospital and Chang Gung University, College of Medicine, Kaohsiung, 83301, Taiwan

${ }^{5}$ Department of Medical Research, China Medical University Hospital, China Medical University, Taichung, 40402, Taiwan

${ }^{6}$ Department of Nursing, Asia University, Taichung, 41354, Taiwan

${ }^{\dagger}$ Author for correspondence: Hon-Kan Yip, Division of Cardiology, Department of Internal Medicine, Kaohsiung Chang Gung Memorial Hospital 123, Dapi Road, Niaosung Dist., Kaohsiung city, 83301, Tel: +886-7-7317123; Fax: +886-7-7322402; email: han.gung@msa. hinet.net
} 


\begin{abstract}
Introduction
Abundant data have shown that carotid revascularization is effective in reducing risk of ischemic stroke (IS) for patients with highgrade carotid artery (CA) stenosis [1-6]. Surgical carotid endarterectomy (CEA) is the first established gold standard treatment for symptomatic CA stenosis [1-4,7,8]. On the other hand, carotid artery stenting (CS) has emerged as a global consensus to be an alternative option for those patients who are at high risk for CEA [5,6,9-11]. However, perioperative neurological complications inevitably occurred in both interventional procedures [4-6,912]. Some clinical observational studies have shown that the rate of periprocedural stroke is notably higher in CS than in CEA $[6,12,13]$. Numerous factors, such as plaque morphology, age, technical expertise and protection devices, have been identified to be related to periprocedural IS [14-18]. Of these factors, plaque characteristics/component identified by virtual histology intravascular ultrasound $(\mathrm{VH}-$ IVUS) [19-21] has been established as one of the paramount factors for risk of periprocedural IS $[20,22]$. However, although to identify the plaque characteristics is one of better strategy for providing useful information for assessing the risk during CS, the CS procedure remains very invasive. Accordingly, to find an alternative method with less invasive and easily performed manner is eager for physicians and patients.
\end{abstract}

Microparticles (MPs), membrane-bound vesicles, are small fragments of the plasma membrane (i.e., ranging in size from $0.1 \mu \mathrm{m}$ to $1.0 \mu \mathrm{m}$ ). Actually, MPs which are released into extracellular space or circulation by direct budding from the plasma membrane are multiple lineages of activated and/or apoptotic cells, including platelets, endothelial cells, granulocytes, monocytes, macrophages, and lymphocytes can mediate inflammation and thrombosis [2329]. Additionally, circulating MPs has recently emerged as a promising biomarker for predicting prognostic outcome of some disease entities [30-32]. Our previous studies have also shown that circulating platelet-derived activated MPs (PDAc-MPs) and endothelial-derived activated MPs (EDAc-MPs) were not only strongly associated with chronic kidney disease [33] and angiogenesis $[34,35]$ but also significantly predictive of prognostic outcome in lung cancer patients [36-38]. Furthermore, leukocytederived MPs has been reported to constitute a promising biomarker associated with plaque vulnerability in patients with high-grade CA stenosis [39]. These aforementioned issues raise [23-39] the hypothesis that circulating MPs may be a useful biomarker with easily obtain by minimal invasive procedure (i.e., to collect the blood sample by need puncture) for an indirect assessment of carotid-plaque characteristics and the possible risk of embolic IS after CS. However, a correlation between the necrotic area of plaque (i.e., an indicator of vulnerable plaque for predictive of embolic event during the procedure) and circulating levels of PDAcMPs and EDAc-MPs has to be confirmed prior to utilize these MPs in our daily clinical practice. Accordingly, this study assessed the necrotic area of carotid plaque by VH-IVUS and circulating levels of PDAc-MPs and EDAc-MPs during the CS procedure.

\section{Materials and Methods \\ - Study design \\ This clinical study was approved by the Institutional Review Committee on Human Research in Chang Gung Memorial Hospital (104-5094C) in 2013 and conducted at Kaohsiung Chang Gung Memorial Hospital. Informed consent was obtained from all study subjects. \\ - Patient population, inclusion and exclusion criteria}

Enrollment and exclusion criteria were based on our previous reports [40-42] with some modification, included the following: 1) in symptomatic patients, extra-cranial carotid artery (ECCA) stenosis (i.e., defined as internal or common carotid artery stenosis $\geq 60 \%$ ) determined by magnetic resonance imaging (MRI)/MR angiography (MRA) and duplex ultrasound of carotid arteries prior to extracranial and intra-cranial angiographic studies; 2 ) history of stroke (> 2 months), transient ischemic attack or dizziness related to a significant ECCA stenosis; 3) In asymptomatic patients, ECCA stenosis was identified to be $>70 \%$ by MRA/ duplex ultrasound of carotid arteries. Exclusion criteria were as follows: 1) history of acute or recent stroke (<2 months), myocardial infarction, and surgery or trauma within the preceding 2 months; 2) unconsciousness or unwillingness to undergo the procedure.

For analytical purpose, the patients were categorized into group 1 (i.e., right CS group) and group 2 (i.e., left $\mathrm{CS}$ group), respectively. 
Link between Systemic and Cerebral Circulatory Levels of Microparticles and Necrosis Area of Stenotic Carotid Artery in the Patients Undergoing Carotid Stenting

\section{- Procedure and protocol of transradial, transbrachial and transfemoral arterial approach for carotid angiographic study and carotid stenting}

The procedure and protocol of transradial artery (TRA) access for coronary artery and carotid artery (CA) angiographic examinations in the same procedure, and carotid artery intervention have been described in details in our previous reports [40-42]. Briefly, a high ( $>5 \mathrm{~cm}$ distance from the wrist) TRA access using a 6-French (Fr) arterial sheath is routinely applied for both diagnostic coronary and carotid angiographic studies in our hospital. However, transbrachial artery (TBA) access for carotid angiographic study is utilized if the patient is taller than 170 $\mathrm{cm}$ or Allen's test results are positive in both hands.

A left or right high TRA (or TBA) approach was selected at the discretion of the primary operator. Following carotid angiographic studies, the guiding catheters were exchanged, i.e., the 6-Fr Kimny guiding catheter was replaced with 7-Fr Kimny guiding catheter (Boston Scientific, Scimed, Inc. Maple Grove, MN.), for subsequent carotid stenting procedure if indicated.

If TRA or TBA approach was not feasible, traditional method of transfemoral artery (TFA) approach was utilized for those patients.

\section{- Blood sampling for microparticle levels in carotid artery and internal jugular vein}

To assess the MP levels of right internal jugular vein (RIJV), femoral vein (FV) puncture was performed by an 18\# puncture needle. A 6-Fr vein sheath was then successfully inserted into the FV after the needle puncture, followed by a 6-Fr pigtail was advanced into the RIJV for blood samplings. The blood samplings were collected from RIJV for two times, i.e., prior to and 5 minutes after CS.

Similarly, blood samplings from the CS treatment site (i.e., CA) were drawn prior to and 5 minutes after $\mathrm{CS}$.

\section{- Categorization of circulating microparticles into four types}

The circulating MPs were categorized into: (1) platelet-derived activated MPs (PDAc-MPs) (CD31 $\left.{ }^{+} \mathrm{CD}_{42 b^{+}} \mathrm{AN}-\mathrm{V}^{-}\right)$and (2) endothelialderived activated MPs (EDAc-MPs) (CD31 ${ }^{+}$ $\left.\mathrm{CD}^{2} \mathrm{~b}^{-} \mathrm{AN}-\mathrm{V}^{-}\right)$based on our previous reports $[33,38]$.

\section{- Blood samples for flow cytometric analysis of microparticle levels}

The procedure and protocol of flow cytometric analysis for determining the MP levels in RIJV (i.e., cerebral circulation) and carotid artery (i.e., systemic circulation) have been described in our previous reports [33,38]. In details, blood samplings were collected in acid citrate dextrose (ACD) vacutainer tubes. The blood sample (1.5 $\mathrm{mL}$ ) was centrifuged at $2500 \mathrm{~g}$ at $4^{\circ} \mathrm{C}$ for $15 \mathrm{~min}$ without acceleration or break to prepare plateletrich plasma. The $250 \mu \mathrm{l}$ plasma samples were thawed and centrifuged for $10 \mathrm{~min}$ at 19,800 $\mathrm{g}$ at $4^{\circ} \mathrm{C}$, and then collected for investigation of microparticles (MPs) smaller than $1.0 \mu \mathrm{m}$.

Size calibration was conducted with $1.0 \mu \mathrm{m}$ beads (Invitrogen, Carlsbad, CA). The MP pellet was re-suspended with $150 \mu \mathrm{l}$ of Annexin-V binding buffer (BD Biosciences). All buffers were sterile-filtered with a $0.2 \mu \mathrm{m}$ filter. The $100 \mu \mathrm{l}$ MPs were then incubated in a TruCOUNT tube (BD Biosciences) with fluorescent monoclonal antibodies: (1) phycoerythrin (PE)-labeled anti-CD31(BD Biosciences); (2) fluorescein isothiocyanate-labeled anti-Annexin-V (BD Biosciences) and; (3) phycoerythrin-Cy5(PECy5)-labeled anti-CD42b (BD Biosciences). The samples were incubated in the dark for 15 min at room temperature. The samples were then analyzed on a FC500 flow cytometer (Beckman Coulter) after $400 \mu \mathrm{l}$ Annexin-V binding buffer was added. The absolute count of MPs was measured setting the stop condition for TruCount beads at 10,000 events.

- The procedure and protocol of virtual histology intravascular ultrasound (vhivus)

After wiring across stenotic lesion and using a filter for distal embolic protection, VH-IVUS (Volcano, Philips) was placed at stenosis for complete lesion assessment. Four different histological components of plaque, i.e., dark green (fibrous), yellow/green (fibrofatty), white (calcified), and red (necrotic lipid core), was immediately provided by VH-IVUS with lumen colored black. Besides, stenosis (\%) was calculated as minimum lumen area (MLA, $\mathrm{mm}^{2}$ ) divided by reference vessel area (RVA, $\mathrm{mm}^{2}$ ).

\section{- Medications}

Patients were pretreated with aspirin $100 \mathrm{mg} /$ day or clopidogrel $75 \mathrm{mg} /$ day orally. Clopidogrel (300 mg loading dose prior to the procedure, then $75 \mathrm{mg} /$ day) was administered to patients 
for at least 3 months following stenting, and aspirin $(100 \mathrm{mg} /$ day) was administered orally to each patient indefinitely.

\section{- Statistical analysis}

Continuous variables with normal distribution were expressed as mean \pm standard deviation, and the difference between two groups was analyzed with independent t-test. Continuous variables which didn't meet normality were reported as median, 25 percentile and 75 percentile, and the difference in groups was examined using MannWhitney U test. Discrete or categorical variables between different groups were reported as percentage and number, and then analyzed with Chi-square test or Fisher's exact test. Because the level of MPs, including different types and changes before and after CS, had big variation and extreme values, we normalized the data by using ratio of post-CS to pre-CS MPs. $\log _{10}$ of the post/pre-CS ratio of MPs were taken after excluding extreme values. The median value of paired samples was analyzed with Wilcoxon's signed-rank test. Additionally, Spearman's rank correlation was adopted to assess the relation between post/pre-CS ratios of MPs and plague necrosis or clinical events, respectively. Furthermore, stepwise multivariate linear regression analysis for different type of MPs was performed with forward selection approach to identify the potential predictive variables. In detail, we defined the post/pre-CS ratio of MPs as response variables, and then use stepwise method to screen important corresponding variables. We processed remaining data with logarithmic transformation. The outlier was then eliminated after normality test. Finally, the study subjects were enrolled for multivariate regression analysis. Statistical analysis was performed using SPSS statistical software for Windows version 22 (SPSS for Windows, version 22; SPSS, IL, U.S.A.). A value of $\mathrm{p}<0.05$ was considered statistically significant.

\section{Results \\ - Baseline characteristics and angiographic results of 92 study subjects}

The age, male gender, body height, body weight and body mass index did not differ between group 1 (i.e., right CS group) and group 2 (left CS group). Additionally, the incidences of smoking, hypertension, diabetes mellitus, hyperlipidemia, peripheral arterial occlusive disease, atrial fibrillation, old stroke, and head and neck cancer with radiation therapy were similar between groups 1 and 2. Furthermore, the prescription of medications, including antiplatelet agents (i.e. aspirin or clopidogrel), angiotensin converting enzyme inhibitors (ACEIs)/angiotensin II type I receptor blockers (ARBs) were also similar between groups 1 and 2 (Table 1 ).

The laboratory findings showed that there were no differences in terms of white blood cell and platelet counts, percentages of segment, lymphocyte, monocyte, eosinophil and basophil, ratio of neutrophil to lymphocyte and ratio of platelet to lymphocyte. Additionally, the serum levels of creatinine, total cholesterol, and lowdensity lipoprotein also did not differ between groups 1 and 2 .

The frequency of TRA/TBA approach for carotid intervention was significantly higher than TFA approach. The coronary artery angiographic examination showed that the rate of concomitant significant coronary and carotid artery stenosis was greater than $74 \%$. Of them, ten percent of CAD patients had left main trunk involvement. However, the frequency of one, two or triple vessel disease and LM disease did not differ between groups 1 and 2 .

The frequency of significant bilateral CA stenosis did not differ between groups 1 and 2. However, the study patients had higher rate of right-side than left-side CA stenosis.

The procedural success rate (i.e., successfully stent implantation) was $100 \%$ in both groups 1 and 2. The incidence of acute ischemic stroke or transient ischemic attack after stenting was acceptable and did not differ between groups 1 and 2 .

The VH-IVUS examination for identifying the obstructive CA lesions and plaque component, and flow cytometric analysis for the level of plasma microparticles to assess the plaque morphological features, burden and stenosis of obstructive CA, VH-IVUS examination was performed prior to CS. The results showed that the percentage of stenosis, minimal lumen area, reference vascular area and component of necrotic tissue did not differ between the groups 1 and (Table 2).

Flow cytometric analysis showed that the blood level of PDAc-MPs in CA (APDMPs) did not differ between groups 1 and 2 prior to and after CS. In addition, the blood level of PDAcMPs in RIJV (VPDMPs) also did not differ between groups 1 and 2 prior to and after CS. 
Link between Systemic and Cerebral Circulatory Levels of Microparticles and Necrosis Area of Stenotic Carotid Artery in the Patients Undergoing Carotid Stenting

Research

\begin{tabular}{|c|c|c|c|c|}
\hline Variables & $\begin{array}{l}\text { Total } \\
(n=92)\end{array}$ & $\begin{array}{l}\text { Right CS } \\
(n=56)\end{array}$ & $\begin{array}{l}\text { Left CS } \\
(n=36)\end{array}$ & P-value \\
\hline \multicolumn{5}{|l|}{ Clinical features } \\
\hline Age (years) & $70.9 \pm 9.8$ & $70.7 \pm 8.8$ & $71.2 \pm 11.3$ & 0.823 \\
\hline Male gender & $82.6 \%(76)$ & $80.4 \%(45)$ & $94.1 \%(31)$ & 0.477 \\
\hline Body height $(\mathrm{cm})$ & $161.6 \pm 6.9$ & $161.3 \pm 7.3$ & $162.1 \pm 6.2$ & 0.611 \\
\hline Body weight (kg) & $63.9 \pm 11.1$ & $63.8 \pm 12.1$ & $64.1 \pm 9.3$ & 0.891 \\
\hline Body mass index $\left(\mathrm{kg} / \mathrm{m}^{2}\right)$ & $24.4 \pm 3.8$ & $24.5 \pm 4.3$ & $24.4 \pm 2.9$ & 0.876 \\
\hline Smoking history & $27.2 \%(25)$ & $33.9 \%(19)$ & $16.7 \%(6)$ & 0.069 \\
\hline Hypertension & $85.7 \%(78)$ & $85.5 \%(47)$ & $86.1 \%(31)$ & 0.930 \\
\hline Diabetes mellitus & $38 \%(35)$ & $39.3 \%(22)$ & $36.1 \%(13)$ & 0.760 \\
\hline Dyslipidemia & $56.5 \%(52)$ & $58.9 \%(33)$ & $52.8 \%(19)$ & 0.561 \\
\hline PAOD & $2.2 \%(2)$ & $3.6 \%(2)$ & $0 \%(0)$ & 0.252 \\
\hline Atrial fibrillation & $6.5 \%(6)$ & $7.1 \%(4)$ & $5.6 \%(2)$ & 0.763 \\
\hline Old stroke & $45.7 \%(42)$ & $44.6 \%(25)$ & $47.2 \%(17)$ & 0.808 \\
\hline $\mathrm{NPC}$ or $\mathrm{H} / \mathrm{N}$ tumor & $16.3 \%(15)$ & $16.1 \%(9)$ & $16.7 \%(6)$ & 0.940 \\
\hline \multicolumn{5}{|l|}{ Medication } \\
\hline Antiplatelet & $100 \%(92)$ & $100 \%(56)$ & $100 \%(36)$ & 1.000 \\
\hline ARB/ACEI & $60.9 \%(56)$ & $69.6 \%(39)$ & $47.2 \%(17)$ & 0.032 \\
\hline Statin & $60.9 \%(56)$ & $55.4 \%(31)$ & $69.4 \%(25)$ & 0.177 \\
\hline \multicolumn{5}{|l|}{ Laboratory data } \\
\hline Leukocyte count $\left(\times 10^{3}\right)$ & $7.3 \pm 2.6$ & $7.4 \pm 2.8$ & $7.2 \pm 2.1$ & 0.700 \\
\hline Segment (\%) & $63.0 \pm 11.9$ & $64.1 \pm 12.5$ & $61.3 \pm 11.1$ & 0.278 \\
\hline Lymphocyte (\%) & $26.4 \pm 8.9$ & $25.6 \pm 8.4$ & $27.6 \pm 9.6$ & 0.303 \\
\hline Monocyte (\%) & $7.9 \pm 11.4$ & $8.8 \pm 14.8$ & $6.6 \pm 1.8$ & 0.274 \\
\hline Eosinophil (\%) & $3.4 \pm 3.3$ & $2.9 \pm 3.2$ & $4.1 \pm 3.3$ & 0.090 \\
\hline Basophil (\%) & $0.3 \pm 0.3$ & $0.3 \pm 0.3$ & $0.4 \pm 0.2$ & 0.599 \\
\hline Platelet count $\left(\times 10^{3}\right)$ & $200.9 \pm 53.7$ & $201.4 \pm 58.0$ & $200.2 \pm 47.1$ & 0.918 \\
\hline NLR & $2.9 \pm 1.9$ & $3.0 \pm 1.5$ & $2.9 \pm 2.3$ & 0.785 \\
\hline PLR & $123.7 \pm 50.8$ & $125.6 \pm 48.4$ & $120.8 \pm 54.7$ & 0.664 \\
\hline Serum creatinine (mg/dL) & $1.2 \pm 0.8$ & $1.2 \pm 0.9$ & $1.2 \pm 0.8$ & 0.853 \\
\hline Total cholesterol (mg/dL) & $152.8 \pm 33.6$ & $150.8 \pm 34.6$ & $156.1 \pm 32.3$ & 0.542 \\
\hline Low density lipoprotein & $88.9 \pm 29.2$ & $89.6 \pm 31.9$ & $88.0 \pm 24.6$ & 0.843 \\
\hline \multicolumn{5}{|l|}{ Procedural information } \\
\hline Right side approach & $75 \%(69)$ & $98.2 \%(55)$ & $38.9 \%(14)$ & $<0.001$ \\
\hline Radial/brachial access & $70.7 \%(65)$ & $73.2 \%(41)$ & $66.7 \%(24)$ & 0.501 \\
\hline Femoral access & $29.3 \%(27)$ & $26.8 \%(15)$ & $33.3 \%(12)$ & 0.501 \\
\hline Coronary angiography & $97.8 \%(90)$ & $98.2 \%(55)$ & $97.2 \%(35)$ & 0.750 \\
\hline CAD & $74.4 \%(67)$ & $78.2 \%(43)$ & $68.6 \%(24)$ & 0.308 \\
\hline Single-vessel CAD & $22.2 \%(20)$ & $20 \%(11)$ & $25.7 \%(9)$ & 0.795 \\
\hline Two-vessel CAD & $18.9 \%(17)$ & $18.2 \%(10)$ & $20 \%(7)$ & 0.830 \\
\hline Triple-vessel CAD & $33.3 \%(30)$ & $40 \%(22)$ & $22.9 \%(8)$ & 0.112 \\
\hline Left main trunk stenosis & $10 \%(9)$ & $7.3 \%(4)$ & $14.3 \%(5)$ & 0.302 \\
\hline Carotid angiography & $100 \%(92)$ & $100 \%(56)$ & $100 \%(36)$ & 1.000 \\
\hline Right CCA stenosis $\geq 50 \%$ & $16.3 \%(15)$ & $23.2 \%(13)$ & $5.6 \%(2)$ & 0.054 \\
\hline Righ ICA stenosis $\geq 50 \%$ & $69.6 \%(64)$ & $92.9 \%(52)$ & $33.3 \%(12)$ & $<0.001$ \\
\hline Left CCA stenosis $\geq 50 \%$ & $10.9 \%(10)$ & $5.4 \%(3)$ & $19.4 \%(7)$ & 0.070 \\
\hline Left ICA stenosis $\geq 50 \%$ & $57.6 \%(53)$ & $41.1 \%(23)$ & $83.3 \%(30)$ & 0.001 \\
\hline Bilateral carotid stenosis & $41.3 \%(38)$ & $42.9 \%(24)$ & $38.9 \%(14)$ & 0.706 \\
\hline \multicolumn{5}{|l|}{ Procedure and clinical outcome } \\
\hline Procedure success rate† & $100 \%(92)$ & $100 \%(56)$ & $100 \%(36)$ & 1.000 \\
\hline Transient ischemic attack & $3.3 \%(3)$ & $3.6 \%(2)$ & $2.8 \%(1)$ & 0.834 \\
\hline Acute embolic stroke‡ & $4.3 \%(4)$ & $5.4 \%(3)$ & $2.8 \%(1)$ & 0.554 \\
\hline Cumulative 1-year mortality & $2.2 \%(2)$ & $3.6 \%(2)$ & $0 \%(0)$ & 0.252 \\
\hline Cumulative 1-year stroke & $6.5 \%(6)$ & $8.9 \%(5)$ & $2.8 \%(1)$ & 0.244 \\
\hline
\end{tabular}




\section{Research Hon-Kan Yip}

Data are expressed as mean $\pm \mathrm{SD}$ or $\%(\mathrm{n})$.

Abbreviation: $\mathrm{CS}$, carotid stenting; PAOD, peripheral arterial occlusive disease; NPC, nasopharyngeal cancer; $\mathrm{H} / \mathrm{N}$, head and neck; $A R B / A C E I$, angiotensin II type I receptor blocker/angiotensin converting enzyme inhibitor; NLR, neutrophil-to-lymphocyte ratio; PLR, platelet-to-lymphocyte ratio; CAD, coronary artery disease; CCA, common carotid artery; ICA, internal carotid artery.

tThe procedure success of carotid stenting is defined as residual $<30 \%$ stenosis on final carotid angiography.

¥Procedure-related stroke within one week after carotid stenting

Continuous and categorical variables between two groups were compared with independent $t$ test and Chi-square test, respectively.

\begin{tabular}{|c|c|c|c|c|}
\hline Variables & $\begin{array}{l}\text { Total } \\
(n=92)\end{array}$ & $\begin{array}{l}\text { Right CS } \\
(n=56)\end{array}$ & $\begin{array}{l}\text { Left CS } \\
(n=36)\end{array}$ & P-value ${ }^{1}$ \\
\hline \multicolumn{5}{|l|}{ Plague on lesion site } \\
\hline Stenosis (\%) & $87.1 \pm 8.9$ & $86.8 \pm 10.7$ & $87.6 \pm 5.7$ & 0.691 \\
\hline Minimum lumen area $\left(\mathrm{mm}^{2}\right)$ & $5.4 \pm 2.4$ & $5.4 \pm 2.4$ & $5.4 \pm 2.4$ & 0.976 \\
\hline Reference vessel area $\left(\mathrm{mm}^{2}\right)$ & $44.7 \pm 6.0$ & $44.9 \pm 5.9$ & $44.4 \pm 6.1$ & 0.734 \\
\hline Necrosis component (\%) & $24.7 \pm 10.2$ & $24.2 \pm 10.9$ & $25.3 \pm 9.1$ & 0.642 \\
\hline \multicolumn{5}{|l|}{ Microparticles } \\
\hline APDMPs-Pre & $\begin{array}{l}84951.2 \\
(39543.5,229764.5)\end{array}$ & $\begin{array}{l}95528.5 \\
(41933.8,231137.3)\end{array}$ & $\begin{array}{l}77480.1 \\
(29844.0,208873.4)\end{array}$ & 0.662 \\
\hline APDMPs-Post & $\begin{array}{l}218473.1 \\
(87788.5,378584.9)\end{array}$ & $\begin{array}{l}207492.0 \\
(74940.8,344341.5)\end{array}$ & $\begin{array}{l}298648.6 \\
(124991.3,413318.8)\end{array}$ & 0.281 \\
\hline Post/Pre-CS ratio† & $2.09(1.17,4.64)$ & $2.10(1.18,4.37)$ & $2.09(0.92,6.35)$ & 0.783 \\
\hline$P_{\text {-value }}^{2}$ & $<0.001$ & & & \\
\hline VPDMPs-Pre & $\begin{array}{l}56796.0 \\
(23956.0,117491.3)\end{array}$ & $\begin{array}{l}69336.1 \\
(35769.3,120343.3)\end{array}$ & $\begin{array}{l}40404.0 \\
(18294.0,119147.9)\end{array}$ & 0.262 \\
\hline VPDMPs-Post & $\begin{array}{l}89355.2 \\
(41792.5,208611.2)\end{array}$ & $\begin{array}{l}75623.8 \\
(40164.2,663220.3)\end{array}$ & $\begin{array}{l}106061.9 \\
(44705.9,208611.2)\end{array}$ & 0.659 \\
\hline Post/Pre-CS ratio & $1.37(0.59,3.90)$ & $1.23(0.49,4.03)$ & $1.23(0.63,3.75)$ & 0.713 \\
\hline P-value ${ }^{2}$ & 0.006 & & & \\
\hline AEDMPs-Pre & $\begin{array}{l}3317.8 \\
(1110.1,10104.1)\end{array}$ & $\begin{array}{l}3229.4 \\
(1637.0,8000.3)\end{array}$ & $\begin{array}{l}3317.8 \\
(697.2,14553.1)\end{array}$ & 0.750 \\
\hline AEDMPs-Post & $\begin{array}{l}5274.3 \\
(1807.8,15263.4)\end{array}$ & $\begin{array}{l}5948.5 \\
(2035.1,18375.8)\end{array}$ & $\begin{array}{l}4739.0 \\
(1663.1,12142.7)\end{array}$ & 0.124 \\
\hline Post/Pre-CS ratio & $1.40(0.67,3.33)$ & $1.39(0.67,3.25)$ & $1.54(0.65,4.70)$ & 0.938 \\
\hline P-value ${ }^{2}$ & 0.003 & & & \\
\hline VEDMPs-Pre & $\begin{array}{l}1553.5 \\
(765.9,5863.6)\end{array}$ & $\begin{array}{l}1985.4 \\
(1003.6,6832.9)\end{array}$ & $\begin{array}{l}1229.8 \\
(594.1,5544.4)\end{array}$ & 0.097 \\
\hline VEDMPs-Post & $\begin{array}{l}2517.8 \\
(709.7,7230.2)\end{array}$ & $\begin{array}{l}2309.8 \\
(856.2,9269.5)\end{array}$ & $\begin{array}{l}3147.6 \\
(612.1,6670.4)\end{array}$ & 0.533 \\
\hline Post/Pre-CS ratio & $0.93(0.50,3.60)$ & $0.92(0.43,3.82)$ & $0.99(0.56,3.85)$ & 0.906 \\
\hline P-value $^{2}$ & 0.391 & & & \\
\hline \multicolumn{5}{|c|}{$\begin{array}{l}\text { Data are expressed as mean } \pm \text { SD or median ( } 25 \text { percentile, } 75 \text { percentile). } \\
\text { Abbreviation: VH-IVUS, virtual histology intravascular ultrasound; MPs, microparticles; CS, carotid stenting; } \\
\text { APDMPs, carotid artery (CA) platelet-derived microparticles; VPDMPs, right internal jugular vein (RIJV) platelet-derived microparticles; AEDMPs, CA } \\
\text { endothelium-derived microparticles; VEDMPs, RIJV endothelium-derived microparticles; } \\
\text { †Post/Pre-CS ratio denotes that the level of MPs after CS is divided by those of MPs prior to CS. } \\
1 \text { Variables were compared between right CS and left CS groups using independent t test or Mann-Whitney U test. } \\
\text { 2Pre-CS and post-CS level of MPs were compared using Wilcoxon sign-rank test. }\end{array}$} \\
\hline
\end{tabular}

Consistently, the blood level of EDAc-MPs in both CA and RIJV (AEDMPs and VEDMPs, respectively) expressed an identical pattern of PDAc-MPs in groups 1 and 2.

The level of these four MPs, i.e., PDAc-MPs and EDAc-MPs in CA and RIJV, increased after carotid stenting. Of note, the ratios of post-CS to pre-CS PDAc-MPs level in both CA and RIJV were significantly increased among the study patients. Besides, the ratio of post-CS to pre-CS EDAc-MPs level was significantly increased in CA, but not significantly elevated in RIJV.

- Comparison of plasma PMs prior to and after CS in both CA and RIJV in 92 study patients

Flow cytometric analysis exhibited that the plasma level of PDAc-MPs in CA was significantly 
Link between Systemic and Cerebral Circulatory Levels of Microparticles and Necrosis Area of Stenotic Carotid Artery in the Patients Undergoing Carotid Stenting

higher after CS than prior to CS. Additionally, this measurement further displayed that the plasma level of PDAc-MPs in RIJV was also significantly higher in the post-CS than pre-CS procedure. Similarly, the plasma level of EDAcMPs in CA was also significantly higher after CS than prior to CS. However, this parameter did not differ in RIJV between the time intervals of post CS and prior to CS. These finding suggest that the CS procedure could predominantly activate PDAc-MPs rather than those of EDAcMPs that may be mainly through activation of platelet activity in circulation (Figure 1)

- Correlation between post/pre-CS ratio of microparticles and percentage of necrotic tissues of stenotic carotid-plague components (Table 3, Figure 2)

To elucidate the correlation between necrotic area of CA stenosis and plasma levels of MPs, Spearman's correlation analysis was utilized. The results showed that Post-CS MPs levels, including PDAc-MPs and EDAc-MPs, in CA were significantly associated with necrotic area in CA stenosis. Additionally, the ratio of post-CS to pre-CS PDAc-MPs level in CA was significantly predictive of an increase in necrotic area of CA stenosis, suggesting that the more component of necrotic lipid core of carotid plague, the higher activation level of PDAc-MP in systemic circulation after carotid stenting.

- Correlation between plasma MPs levels and necrotic area and combined one-year cumulative clinical events (Table 4)

To determine the correlation between one-year cumulative combined clinical events (defined as transient ischemic attack, acute IS after CS, IS, or death identified during clinical follow-up) and necrotic area of CA and plasma levels of MPs, we performed Spearman's correlation analysis. The results showed no significant correlation between these parameters.

\section{- Multiple linear regression analysis of predictors for predictive of the ratio of pre-CS to post-CS plasma MPs levels in CA and RIJV (Table 5).}

The multiple regression analysis demonstrated that age was significantly predictive of ratio of post-CS/pre-CS PDAc-MPs and EDAc-MPs levels in CA. Male gender was significantly negatively predictive of the ratio of post-CS/ pre-CS PDAc-MPs and EDAc-MPs levels in CA and positively predictive of the ratio of post-CS/ pre-CS EDAc-MPs level in RIJV. On the other hand, diabetes mellitus was weakly predictive of the ratio of post-CS/pre-CS EDAc-MPs level in RIJV.

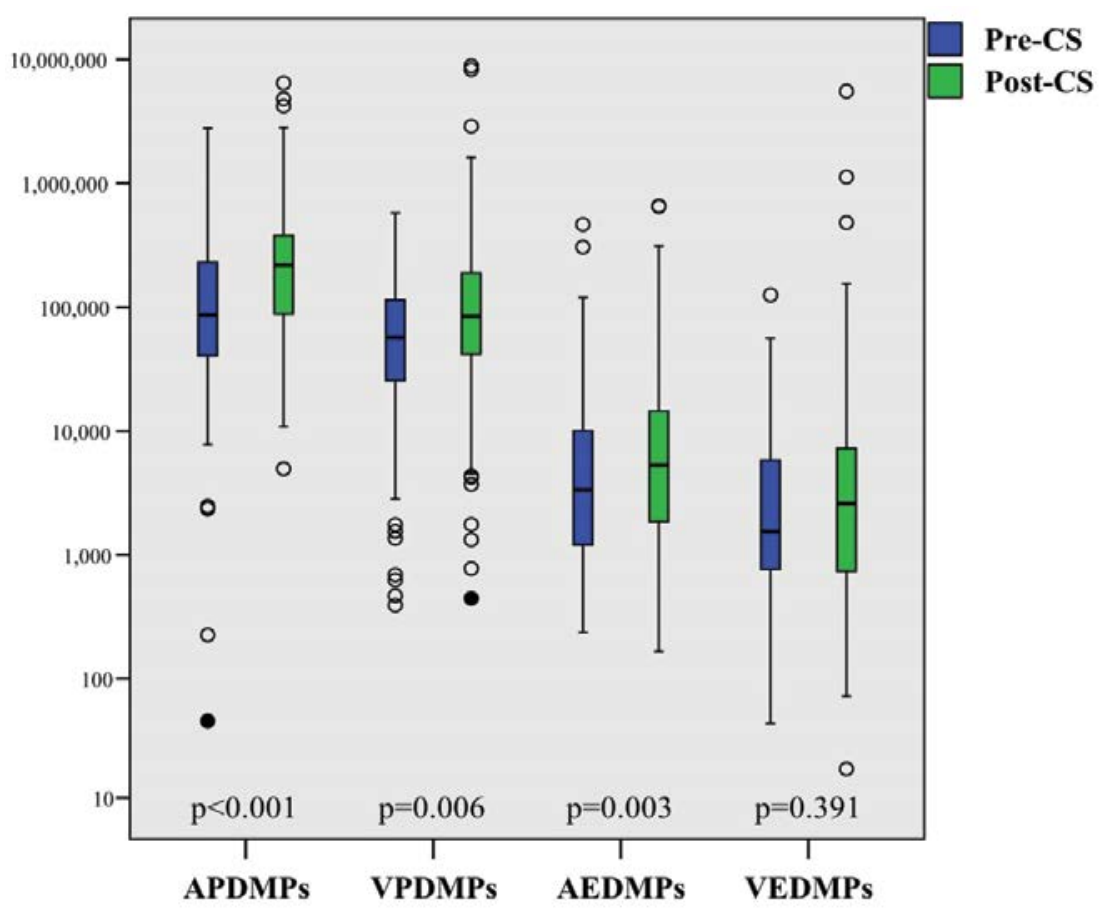

Figure 1: Comparison of the difference of plasma microparticles prior to vs. post carotid stenting (CS) in carotid artery and right internal jugular vein (RIJV).

(1) For platelet-derived activated microparticles in carotid artery (APDMPs), pre-CS vs. post CS, $p<0.001$. (2) For platelet-derived microparticles in RIJV (VPDMPs), $p=0.006$. (3) For endothelialderived activated microparticles in carotid artery (AEDMPs), $p=0.003$. (4) For endothelial-derived activated microparticles in RIJV (VEDMPs), $p=0.391$.

\begin{tabular}{|c|c|c|}
\hline Variables & $\mathbf{R}$ & P-value \\
\hline APDMPs-Pre & -0.070 & 0.594 \\
\hline APDMPs-Post & 0.395 & $<0.001$ \\
\hline Post/Pre-CS ratio of APDMPs & 0.465 & $<0.001$ \\
\hline VPDMPs-Pre & 0.111 & 0.359 \\
\hline VPDMPs-Post & 0.047 & 0.697 \\
\hline Post/Pre-CS ratio of VPDMPs & 0.022 & 0.858 \\
\hline AEDMPs-Pre & 0.062 & 0.603 \\
\hline AEDMPs-Post & 0.312 & 0.010 \\
\hline Post/Pre-CS ratio of AEDMPs & 0.133 & 0.287 \\
\hline VEDMPs-Pre & 0.236 & 0.053 \\
\hline VEDMPs-Post & 0.107 & 0.370 \\
\hline Post/Pre-CS ratio of VEDMPs & -0.134 & 0.277 \\
\hline NLR & 0.185 & 0.112 \\
\hline PLR & 0.171 & 0.141 \\
\hline \multicolumn{3}{|c|}{$\begin{array}{l}\text { Abbreviation: CS, carotid stenting; MPs, microparticles; NLR, neutrophil-to-lymphocyte ratio; } \\
\text { PLR, platelet-to-lymphocyte ratio; R, Spearman's correlation coefficient; APDMPs, carotid } \\
\text { artery (CA) platelet-derived microparticles; VPDMPs, right internal jugular vein (RIJV) platelet- } \\
\text { derived microparticles; AEDMPs, CA endothelium-derived microparticles; VEDMPs, RIJV } \\
\text { endothelium-derived microparticles; } \\
\text { Statistics was done with Spearman's rank correlation. }\end{array}$} \\
\hline
\end{tabular}


Research Hon-Kan Yip
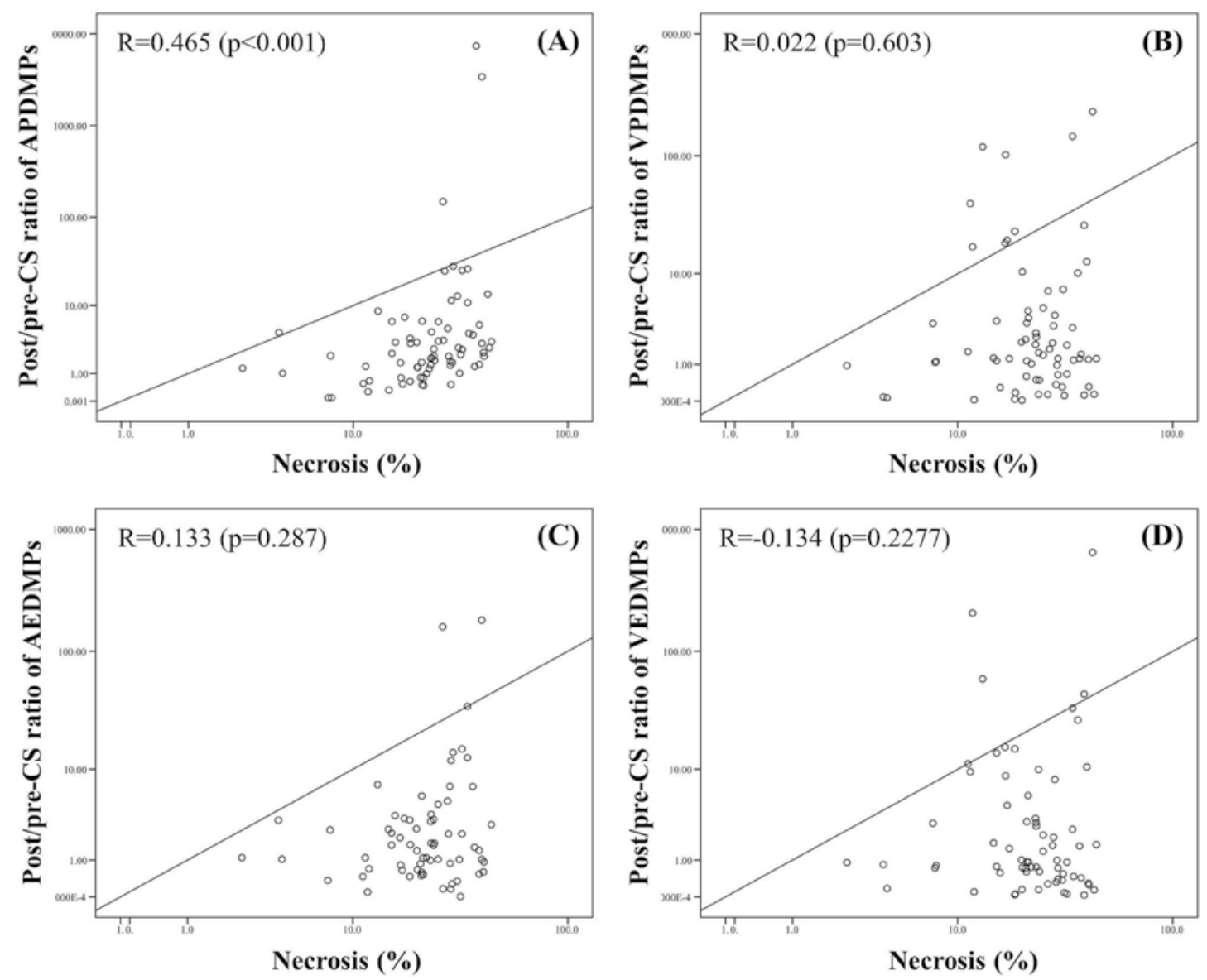

Figure 2: Correlation between the ratio of post-carotid stenting (CS) to pre-CS microparticles (MPs) in carotid artery (CA) and right internal jugular vein (RIJV) and necrosis of carotid plague.

A) Moderate correlation between the ratio of post-CS to pre-CS APDMPs and necrosis area (expressed as $\%), p<0.001$. APDMPs $=$ platelet-derived activated microparticles in CA. B) No correlation between the ratio of post-CS to pre-CS VPDMPs and necrosis area, $p=0.603$. VPDMPs $=$ plateletderived activated microparticles in RIJV. C) No correlation between the ratio of post-CS to pre-CS AEDMPs and necrosis area, $p=0.287$. AEDMPs $=$ endothelial-derived activated microparticles in CA. D) No correlation between ratio of post-CS to pre-CS VEDMPs and necrosis area, $p=0.228$. VEDMPs $=$ endothelial-derived activated microparticles in RIJV.

Table 4: Correlation of necrosis, post/pre-CS ratio of MPs, PLR and NLR to cumulative one-year clinical events

\begin{tabular}{|l|l|l|}
\hline Variables & R & P-value \\
\hline Necrosis & -0.146 & 0.207 \\
\hline Post/Pre-CS ratio of APDMPs & -0.098 & 0.359 \\
\hline Post/Pre-CS ratio of VPDMPs & 0.037 & 0.736 \\
\hline Post/Pre-CS ratio of AEDMPs & 0.072 & 0.522 \\
\hline Post/Pre-CS ratio of VEDMPs & 0.020 & 0.855 \\
\hline $\begin{array}{l}\text { Abbreviation: CS, carotid stenting; MPs, microparticles; R, Spearman's correlation } \\
\text { coefficient; APDMPs, carotid artery (CA) platelet-derived microparticles; VPDMPs, right } \\
\text { internal jugular vein (RIJV) platelet-derived microparticles; } \\
\text { derived microparticles; VEDMPs, RIJV endothelium-derived microparticles; Statistics was } \\
\text { done with Spearman's rank correlation. }\end{array}$ \\
\hline
\end{tabular}

Additionally, prescription of ACEI/ARB was significantly but negatively predictive of ratio of post-CS/pre-CS EDAc-MPs level in CA. Furthermore, bilateral CA stenosis was significantly negatively predictive of post-CS/
pre-CS PDAc-MPs and EDAc-MPs level in RIJV. Moreover, the necrotic tissue was the most strongly significantly predictive of the ratio of PDAc-MPs and EDAc-MPs levels in CA.

\section{Discussion}

This study investigated the correlation between the percentage of necrotic area in CA stenosis and blood plasma level of MPs yielded several striking implications. First, the plasma level of PDAc-MPs in CA and RIJV was significantly higher in post-CS than in pre-CS time point, highlighting that the CS procedure would augment the generation of PDAc-MPs. Second, a moderate correlation existed between ratio of post-CAS to pre-CAS PDAc-MPs levels in CA and necrotic area of CA stenosis. Third, multiple linear regression demonstrated that the necrotic 
Link between Systemic and Cerebral Circulatory Levels of Microparticles and Necrosis Area of Stenotic Research Carotid Artery in the Patients Undergoing Carotid Stenting

\begin{tabular}{|c|c|c|c|c|c|c|c|c|}
\hline \multirow[t]{2}{*}{ Post/pre-CS ratio } & \multicolumn{2}{|l|}{ ADPMPs } & \multicolumn{2}{|l|}{ VPDMPs } & \multicolumn{2}{|l|}{ AEDMPs } & \multicolumn{2}{|l|}{ VEDMPs } \\
\hline & $\begin{array}{l}\text { Coefficient } \\
95 \% \mathrm{Cl}\end{array}$ & P-value & $\begin{array}{l}\text { Coefficient } \\
95 \% \mathrm{Cl}\end{array}$ & P-value & $\begin{array}{l}\text { Coefficient } \\
95 \% \mathrm{Cl}\end{array}$ & P-value & $\begin{array}{l}\text { Coefficient } \\
95 \% \mathrm{Cl}\end{array}$ & P-value \\
\hline Age & $\begin{array}{l}0.04 \\
(0.01,0.06)\end{array}$ & 0.006 & -0.13 & 0.347 & $\begin{array}{l}0.03 \\
(0.006,0.06)\end{array}$ & 0.017 & -0.15 & 0.224 \\
\hline Male gender & $\begin{array}{l}-1.23 \\
(-2.06,-0.4)\end{array}$ & 0.004 & 0.23 & 0.084 & $\begin{array}{l}-1.48 \\
(-2.34,-0.63)\end{array}$ & 0.001 & $\begin{array}{l}1.73 \\
(0.19,3.28)\end{array}$ & 0.029 \\
\hline Body mass index & -0.06 & 0.626 & -0.08 & 0.529 & -0.11 & 0.365 & -0.08 & 0.537 \\
\hline Smoking history & -0.13 & 0.249 & 0.17 & 0.203 & -0.13 & 0.284 & 0.05 & 0.723 \\
\hline Hypertension & -0.04 & 0.756 & -0.25 & 0.058 & -0.11 & 0.371 & -0.05 & 0.729 \\
\hline Diabetes mellitus & 0.02 & 0.876 & -0.19 & 0.138 & 0.16 & 0.184 & $\begin{array}{l}-1.02 \\
(-1.99,-0.04)\end{array}$ & 0.042 \\
\hline Dyslipidemia & 0.02 & 0.838 & 0.11 & 0.395 & 0.11 & 0.371 & 0.08 & 0.536 \\
\hline PAOD & 0.08 & 0.530 & -0.05 & 0.717 & 0.01 & 0.969 & 0.15 & 0.271 \\
\hline Atrial fibrillation & 0.06 & 0.625 & -0.01 & 0.939 & 0.07 & 0.571 & 0.06 & 0.645 \\
\hline Old stroke & 0.08 & 0.506 & 0.08 & 0.939 & -0.01 & 0.928 & -0.03 & 0.829 \\
\hline NPC or $\mathrm{H} / \mathrm{N}$ tumor & 0.04 & 0.732 & -0.08 & 0.544 & 0.08 & 0.541 & -0.003 & 0.983 \\
\hline ACEI/ARB & -0.13 & 0.248 & -0.18 & 0.168 & $\begin{array}{l}-0.70 \\
(-1.24,-0.17)\end{array}$ & 0.011 & -0.11 & 0.376 \\
\hline Statin & -0.16 & 0.186 & -0.12 & 0.377 & -0.02 & 0.866 & 0.05 & 0.728 \\
\hline NLR & -0.06 & 0.610 & -0.21 & 0.100 & 0.06 & 0.631 & -0.11 & 0.376 \\
\hline PLR & -0.18 & 0.121 & -0.23 & 0.078 & -0.17 & 0.146 & -0.12 & 0.337 \\
\hline Serum Creatinine & -0.19 & 0.101 & -0.03 & 0.798 & -0.18 & 0.139 & -0.06 & 0.633 \\
\hline CAD & -0.03 & 0.782 & -0.07 & 0.611 & 0.10 & 0.400 & -0.11 & 0.404 \\
\hline Multi-vessel CAD‡ & -0.06 & 0.624 & -0.16 & 0.216 & 0.03 & 0.801 & -0.18 & 0.146 \\
\hline Bilateral CAS & 0.02 & 0.891 & $\begin{array}{l}-1.29 \\
(-2.38,-0.21)\end{array}$ & 0.021 & -0.17 & 0.158 & $\begin{array}{l}-1.52 \\
(-2.52,-0.52)\end{array}$ & 0.004 \\
\hline Necrosis & $\begin{array}{l}0.05 \\
(0.03,0.08)\end{array}$ & $<0.001$ & -0.03 & 0.803 & $\begin{array}{l}0.04 \\
(0.01,0.06)\end{array}$ & 0.011 & -0.13 & 0.299 \\
\hline \multicolumn{9}{|c|}{$\begin{array}{l}\text { Data are expressed as coefficient ( } 95 \% \mathrm{Cl} \text { was only shown if statistically significant). } \\
\text { Abbreviation: } \mathrm{CS} \text {, carotid stenting; } \mathrm{MP} \text {, microparticles; } \mathrm{Cl} \text {, confidence interval; PAOD, peripheral arterial occlusive disease; NPC, nasopharyngeal cancer; } \\
\text { H/N, head and neck; ARB/ACEI, angiotensin II type I receptor blocker/angiotensin converting enzyme inhibitor; NLR, neutrophil-to-lymphocyte ratio; PLR, } \\
\text { platelet-to-lymphocyte ratio; CAD, coronary artery disease; CAS, carotid artery stenosis. } \\
\text { APDMPs, carotid artery (CA) platelet-derived microparticles; VPDMPs, right internal jugular vein (RIJV) platelet-derived microparticles; AEDMPs, CA } \\
\text { endothelium-derived microparticles; VEDMPs, RIJV endothelium-derived microparticles; } \\
\text { *Stepwise multivariate linear regression analysis was done with adjusting for age, gender, comorbidities, medication, and lesion characteristics. } \\
\text { †Post/Pre-CS ratio denotes that the level of MPs after CS is divided by those of MPs prior to CS. } \\
\text { †Multi-vessel CAD indicated two-vessel and triple-vessel CAD. }\end{array}$} \\
\hline
\end{tabular}

area was significantly predictive of the ratio of post-CAS to pre-CAS PDAc-MPs and EDAcMPs level in CA.

Our previous studies have shown the frequency of significant $\mathrm{CAD}$ in patients with high-grade CA stenosis was more than $75 \%[40,41,43]$. One important finding in the present study was that the frequency of significant $\mathrm{CAD}$ in $\mathrm{CS}$ patients was more than $74 \%$. Additionally, the rate of significant stenosis of LM trunk was $10 \%$. Our findings, consistent with our previous studies $[40,41,43]$, imply that CAD and CA stenosis are the two sides of the same coin, resulting from endothelial dysfunction, plaque formation, and arterial obstructive syndrome. Importantly, our findings strongly recommend that carotid and coronary arterial angiographic studies should be simultaneously examined in one stage for categorizing the CA stenosis patients into the high-risk and low-risk subgroups.

Platelet reactivity has been established as a fundamental role in the pathogenesis of atherosclerosis and thromboembolic events [44-46]. Our previous study has further demonstrated that the platelet activity in RIJV (i.e., cerebral circulation) and femoral vein (i.e., systemic circulation) was significantly increased in high-grade CA stenosis patients than in those of at risk control subjects [47]. Additionally, as compared with prior CS, the platelet activity was still persistently or more activated in patients after CS [47]. Intriguingly, our other previous studies have also displayed that the circulating level of soluble CD40 ligand (sCD40L), an index of platelet activation, was significantly higher in patients with coronary arterial 
obstructive syndrome undergoing coronary stenting [48-50]. Additionally, sCD40L was predictive of angiographic morphologic features of high-burden thrombus formation [50] that is well known to play a crucial role for procedural failure during coronary intervention and unfavorable prognostic outcome. A principal finding in the present study was that PDAc-MPs was remarkably higher in the time point of postCS than pre-CS procedure. Additionally, this phenomenon was not only identified in systemic circulation but also in the cerebral circulation, highlighting that the CS procedure would frequently induce an upregulation of PDAc-MPs even undergoing the loading dose of double antiplatelet agents (i.e., aspirin and clopidogrel). Our findings, comparable with our previous studies [47-50], encourage the use of circulating MPs as an alternatively accessory modality for assessing the platelet activity in patients with arterial obstructive syndrome.

It is well recognized that vulnerable plaque usually contains high lipid-core content which is crucial for no-reflow phenomenon during coronary intervention. The acute no-/slowflow phenomenon always results from the dislodged lipid-core content/debris to plug the microcirculation and results in an unfavorable prognostic outcome. Interestingly, previous studies have shown that leukocyte-derived and endothelial-derived MPs are the promising biomarkers associated with plaque vulnerability in patients with significant CA stenosis [39,51]. In the present study, we found that post-CS PDAc-MPs, EDAc-MPs and ratio of post-CS/ pre-CS EDAc-MPs levels (Table 3 and Figure 2) were strongly and significantly correlated to necrotic area of CA stenosis. In this way, our finding, in addition to the findings of previous studies [39,51], imply that circulating MPs could be useful non-invasive derived biomarker for predicting the vulnerable plaque not only in patients with CA stenosis but also in those of patients with other arterial obstructive syndromes.

The aforementioned aims of the study in introduction section were (1) to find whether the cerebral/systemic circulation of MPs could be a useful biomarker for predictive of vulnerable plaque in CS stenosis and (2) to assess whether the cerebral/systemic circulation of MPs would be useful biomarker for predictive of embolic IS in patients after receiving CS. The result of $2^{\text {nd }}$ study aim might be disappointing because there was no significant correlation between ratios of post/pre-CS MPs in both CA and RIJV (Table 4). Intriguingly, previous study has found that leukocyte-derived MPs was strongly associated with at risk of thromboembolic and neurologic events in patients with asymptomatic CA stenosis undergoing the endarterectomy [39]. In this way, the results of our and previous study [39] is inconsistent. Perhaps, several reasons could be explained the discrepancy between our results and findings of previous study [39]. First, the carotid intervention procedure was different between these two studies, i.e., CS vs. carotid endarterectomy. Second, filter protection was used for preventing distal embolization in our study but not in the previous study [39]. Third, the measured type of MPs differed between our and previous studies [39].

\section{Study Limitations}

This study has limitations. Frist, the patient sample size was still relatively small and the standard deviation of MPs level was extremely high. These phenomena may distort the statistical significance. Second, no correlation between oneyear cumulatively untoward clinical outcomes and necrotic area and circulating MPs level could be perhaps due to the extremely highly standard deviation of MPs level.

\section{Conclusion}

In conclusion, plasma levels of PDAc-MPs and EDAc-MPs were remarkably increased in the post-CS than pre-CS procedure. The results of the present study displayed that post-CS PDAc-MPs/EDAc-MPs levels and ratio of post/pre-CS PDAc-MPs were significantly correlated to necrotic area of CA stenosis. However, the results of the present study did not show circulating MPs or necrotic area is a useful predictor for one-year cumulative combined clinical events. 
Link between Systemic and Cerebral Circulatory Levels of Microparticles and Necrosis Area of Stenotic Research Carotid Artery in the Patients Undergoing Carotid Stenting

\section{References}

1. Barnett HJM, Taylor DW, Haynes RB, et al. Beneficial effect of carotid endarterectomy in symptomatic patients with high-grade carotid stenosis. N. Engl. J. Med 325(7), 445-453 (1991).

2. Barnett HJ, Taylor DW, Eliasziw M, et al. Benefit of carotid endarterectomy in patients with symptomatic moderate or severe stenosis. North American Symptomatic Carotid Endarterectomy Trial Collaborators. N. Engl. J. Med 339(20),1415-1425 (1998).

3. Mayberg MR, Wilson SE, Yatsu F, et al. Carotid endarterectomy and prevention of cerebral ischemia in symptomatic carotid stenosis. Veterans Affairs Cooperative Studies Program 309 Trialist Group. JAMA 266(23), 3289-3294 (1991).

4. Randomised trial of endarterectomy for recently symptomatic carotid stenosis: final results of the MRC European Carotid Surgery Trial (ECST). Lancet (London, England) 351(9113),1379-1387 (1998).

5. Yadav JS, Wholey MH, Kuntz RE, et al. Protected carotid-artery stenting versus endarterectomy in high-risk patients. N. Engl. J. Med 351(15),1493-1501 (2004).

6. Brott TG, Hobson RW, 2nd, Howard G, et al. Stenting versus endarterectomy for treatment of carotid-artery stenosis. N. Engl. J. Med 363(1), 11-23 (2010).

7. Ferguson GG, Eliasziw M, Barr HW, et al. The North American Symptomatic Carotid Endarterectomy Trial : surgical results in 1415 patients. Stroke 30(9), 1751-1758 (1999).

8. Young B, Moore WS, Robertson JT, et al. An analysis of perioperative surgical mortality and morbidity in the asymptomatic carotid atherosclerosis study. ACAS Investigators. Asymptomatic Carotid Atherosclerosis Study. Stroke 27(12), 2216-2224 (1996).

9. Roubin GS, New G, lyer SS, et al. Immediate and late clinical outcomes of carotid artery stenting in patients with symptomatic and asymptomatic carotid artery stenosis: a 5-year prospective analysis. Circulation 103(4), 532537 (2001).

10. Endovascular versus surgical treatment in patients with carotid stenosis in the Carotid and Vertebral Artery Transluminal Angioplasty Study (CAVATAS): a randomised trial. Lancet (London, England) 357(9270), 1729-1737 (2001).

11. Bonati LH, Dobson J, Featherstone RL, et al. Long-term outcomes after stenting versus endarterectomy for treatment of symptomatic carotid stenosis: the International Carotid Stenting Study (ICSS) randomised trial. Lancet (London, England) 385(9967), 529-538 (2015).

12. Ouriel K, Hertzer NR, Beven EG, et al. Preprocedural risk stratification: identifying an appropriate population for carotid stenting. J. of. Vasc. Surgery 33(4), 728-732 (2001).

13. Rosenfield K, Matsumura JS, Chaturvedi S, et al. Randomized Trial of Stent versus Surgery for Asymptomatic Carotid Stenosis. N. Engl. J. Med 374(11), 1011-1020 (2016).

14. Hobson RW, 2nd, Howard VJ, Roubin GS, et al. Carotid artery stenting is associated with increased complications in octogenarians: 30-day stroke and death rates in the CREST lead-in phase. J. Vasc. Surgery 40(6), 11061111 (2004).

15. Bosiers M, de Donato G, Deloose K, et al. Does free cell area influence the outcome in carotid artery stenting? European journal of vascular and endovascular surgery : the official journal of the European. Society. For. Vascular. Surgery 33(2), 135-141 discussion 142-133 (2007).

16. Jansen O, Fiehler J, Hartmann M, et al. Protection or nonprotection in carotid stent angioplasty: the influence of interventional techniques on outcome data from the SPACE Trial. Stroke 40(3), 841-846 (2009).

17. Sakamoto $M$, Taoka T, Nakagawa $H$, et al. Magnetic resonance plaque imaging to predict the occurrence of the slow-flow phenomenon in carotid artery stenting procedures. Neuroradiology 52(4), 275-283 (2010).

18. Yoshimura S, Yamada K, Kawasaki M, et al. High-intensity signal on time-of-flight magnetic resonance angiography indicates carotid plaques at high risk for cerebral embolism during stenting. Stroke 42(11), 3132-3137 (2011).

19. Kotsugi M, Takayama K, Myouchin K, et al. Carotid Artery Stenting: Investigation of Plaque Protrusion Incidence and Prognosis. JACC. Cardiovascular. Interventions 10(8), 824831 (2017).

20. Hitchner E, Zhou W. Utilization of Intravascular Ultrasound during Carotid Artery Stenting. The International Journal of Angiology: Official Publication of the International College of Angiology, Inc 24(3),185-188 (2015).

21. Gonzalez A, Lopez-Rueda A, Gutierrez I, et al. Carotid plaque characterization by virtual histology intravascular ultrasound related to the timing of carotid intervention. JEVT 19(6), 764-773 (2012).

22. Wehman JC, Holmes DR, Ecker RD, et al. Intravascular ultrasound identification of intraluminal embolic plaque material during carotid angioplasty with stenting. $\mathrm{CACl} 68(6)$, 853-857 (2006).

23. Horstman LL, Ahn YS. Platelet microparticles: a wide-angle perspective. Crit. Rev. Oncol. Hematol 30(2), 111-142 (1999).

24. Martin S, Tesse A, Hugel B, et al. Shed membrane particles from $T$ lymphocytes impair endothelial function and regulate endothelial protein expression. Circulation
109(13),1653-1659 (2004).

25. Martinez MC, Larbret F, Zobairi F, et al. Transfer of differentiation signal by membrane microvesicles harboring hedgehog morphogens. Blood 108(9), 30123020 (2006).

26. Martinez MC, Tesse A, Zobairi F, et al. Shed membrane microparticles from circulating and vascular cells in regulating vascular function. Am J Physiol Heart Circ Physiol 288(3), H1004-1009 (2005).

27. Mezentsev A, Merks RM, O'Riordan E, et al. Endothelial microparticles affect angiogenesis in vitro: role of oxidative stress. Am. J. Physiol. Heart. Circ. Physiol 289(3), H1106-1114 (2005).

28. Nomura S. Function and clinical significance of platelet-derived microparticles. Int. J. Hematol 74(4), 397-404 (2001).

29. Nomura S, Ozaki Y, Ikeda Y. Function and role of microparticles in various clinical settings. Thrombosis. Research 123(1), 8-23 (2008).

30. Lacroix R, Dignat-George F. Microparticles: new protagonists in pericellular and intravascular proteolysis. Semi. In. Thrombosis. Hemostasis 39(1), 33-39 (2013).

31. Schiro A, Wilkinson FL, Weston R, et al. Endothelial microparticles as conveyors of information in atherosclerotic disease. Atherosclerosis 234(2), 295-302 (2014).

32. Dalli J, Montero-Melendez T, Norling LV, et al. Heterogeneity in neutrophil microparticles reveals distinct proteome and functional properties. Mol. Cell. Proteomics. MCP 12(8), 2205-2219 (2013).

33. Chen YL, Chen CH, Wallace CG, et al. Levels of circulating microparticles in patients with chronic cardiorenal disease. J. Atheroscler. Thromb 22(3), 247-256 (2015).

34. Sheu JJ, Lee FY, Wallace CG, et al. Administered circulating microparticles derived from lung cancer patients markedly improved angiogenesis, blood flow and ischemic recovery in rat critical limb ischemia. J. Clin. Transl. Sci 13(1), 59 (2015).

35. Ko SF, Hsu SY, Chen $\mathrm{CH}$, et al. Human lung cancer-derived microparticles enhanced angiogenesis and growth of hepatoma cells in rodent lung parenchyma. \Am. J. Transl. Res 8(3), 1302-1318 (2016).

36. Tseng CC, Wang CC, Chang HC, et al. Levels of circulating microparticles in lung cancer patients and possible prognostic value. Disease. Markers 35(5), 301-310 (2013).

37. Wang CC, Tseng CC, Hsiao CC, et al. Circulating endothelial-derived activated microparticle: a useful biomarker for predicting one-year mortality in patients with advanced non-small cell lung cancer. BioMed. Research. International 173-401 2014.

38. Tseng CC, Wang CC, Hsiao CC, et al. Time courses and value of circulating 


\section{Research Hon-Kan Yip}

microparticles in patients with operable stage non-small cell lung cancer undergoing surgical intervention. Tumour. Biol 37(9), 11873-11882 (2016).

39. Sarlon-Bartoli G, Bennis Y, Lacroix R, et al. Plasmatic level of leukocyte-derived microparticles is associated with unstable plaque in asymptomatic patients with highgrade carotid stenosis. JACC 62(16), 14361441 (2013).

40. Wu CJ, Hung WC, Chen SM, et al. Feasibility and safety of transradial artery approach for selective cerebral angiography. Catheter. Cardiovasc. Interv 66(1), 21-26 (2005).

41. Wu CJ, Cheng Cl, Hung WC, et al. Feasibility and safety of transbrachial approach for patients with severe carotid artery stenosis undergoing stenting. Catheter. Cardiovasc. Interv 67(6), 967-971 (2006).

42. Yip HK, Sung PH, Wu CJ, et al. Carotid stenting and endarterectomy. Int. J. Cardiol 214(1), 166-174 (2016).

43. Fang HY, Chung SY, Sun CK, et al. Transradial and transbrachial arterial approach for simultaneous carotid angiographic examination and stenting using catheter looping and retrograde engagement technique. Annals. Of. vascular. Surgery 24(5), 670-679 (2010).

44. Fisher M, Francis R. Altered coagulation in cerebral ischemia. Platelet, thrombin, and plasmin activity. Archives. Of. Neurology 47(10), 1075-1079 (1990).

45. Yip HK, Chen SS, Liu JS, et al. Serial changes in platelet activation in patients after ischemic stroke: role of pharmacodynamic modulation. Stroke 35(7), 1683-1687 (2004).

46. Yip HK, Liou CW, Chang HW, et al. Link between platelet activity and outcomes after an ischemic stroke. Cerebrovascular.s Diseases (Basel, Switzerland) 20(2), 120-128 (2005).

47. Yip HK, Lu CH, Yang CH, et al. Levels and value of platelet activity in patients with severe internal carotid artery stenosis. Neurology 2006;66(6):804-808.

48. Yip HK, Wu CJ, Yang CH, et al. Serial changes in circulating concentrations of soluble
CD40 ligand and C-reactive protein in patients with unstable angina undergoing coronary stenting. Circulation. Journal 69(8), 890-895 (2005).

49. Yip HK, Chang LT, Sun CK, et al. Impact of clopidogrel on suppression of circulating levels of soluble CD40 ligand in patients with unstable angina undergoing coronary stenting. Am. J. Car. Res 97(2), 192-194 (2006)

50. Youssef AA, Chang LT, Sheu JJ, et al. Association between circulating level of CD40 ligand and angiographic morphologic features indicating highburden thrombus formation in patients with acute myocardial infarction undergoing primary coronary intervention. Circulation. Journal 71(12), 1857-1861 (2007).

51. Schiro A, Wilkinson FL, Weston $R$, et al. Elevated levels of endothelial-derived microparticles, and serum CXCL9 and SCGF-beta are associated with unstable asymptomatic carotid plaques. Scientific. Reports 5(1), 16658 (2015). 